\title{
Aesthetic Rehabilitation with Ceramic Laminates By the System CAD/CAM- A Case Report
}

\author{
Montenegro G*, Tine JCNP, Silva WO and Pinto T \\ Brazilian Dentistry Association, Brazil
}

Submission: May 17, 2018; Published: August 29, 2018

*Corresponding author: Gil Montenegro,Postgraduate Teacher of Brazilian Dentistry Association, ABO Dental College, Brazil, Email: prof.gilmontenegro@gmail.com

\begin{abstract}
The demand for cosmetic treatments has increased considerably in dental offices.Current laboratory techniques associated with a strict clinical protocol satisfy patients restorative and esthetic needs.Thin ceramic laminates are a suitable option to correct shape, symmetry, contour, and size of anterior teeth. Lithium disilicate (LD) ceramic laminates are excellent treatment options for performing aesthetic rehabilitation due to the material's strength and tensile properties. In this case report, ceramic laminates were fabricated using an all-digital workflow. The case describes the steps involved in the clinical protocol to perform a high-performance treatment with the CEREC (c) CAD /CAM system computeraided design and computer-aided manufacturing, with ceramic laminates.
\end{abstract}

Keywords: CAD/CAM; Ceramic; Esthetic;Laminates

\section{Introduction}

In the last decade there has been a notable advance in the technological issues aimed at the manufacture of prosthetic restorations aiming at optimizing the precision and quality that the patient seeks [1]. Dental rehabilitations such as ceramic laminates, seek to recover patients' oral function for the benefit of their health and aesthetics.The ceramic laminates are suitable systems for aesthetic and functional restoration, mainly because they present principles of biomimetics, that is, the tooth that receives the ceramic restoration behaves functionally as a healthy tooth, in relation to the distribution of the stresses [2].

To produce a good prosthetic restauration and providing natural results in rehabilitations, the study of aesthetic analysis and the development of skills and knowledge in anatomy and dental morphology should be objects of improvement to be applied in conjunction with technological resources $[3,4]$.

With the CEREC $®$ system, it was possible to combine beauty, comfort, precision and speed in dental prostheses. In addition to adding value to users and safety to results, since the system eliminates many steps in the traditional process of prosthetic preparation, such as molding, measurements and use of temporary in some specific situations [5].

The bio generic CAD/CAM program allows the operator to design a restoration through 3 techniques. First, in the individual bigeneric, the neighboring teeth are analyzed, and the restoration is calculated based on this information [6]. A complete image of at least one neighboring tooth should be captured in the occlusal/ incisal direction. Second, in the biogeneric copy/Correlation the tooth to be restored presents a well-preserved occlusal surface or presents a provisional with excellent anatomy, so the program has copied the morphology of the tooth before being prepared. Finally, in the biogeneric reference, the program uses the anatomy of the homologous tooth of another quadrant and mirrors the tooth to be prepared, if we are going to prepare the tooth 14 one can copy the morphology of the tooth 24 and adapt it to the tooth [7].

After the scanning, gets the digital model. Then the program allows to delimit the cervical terminus, to realize the stamping and to allow the exposure of the area to be worked. Other software also makes it possible to draw the piece itself [8]. The design of crowns is originated by a database present in the program, which also provides adjustment tools, so the operator can change the part, if desired, by customizing the work. These programs are very agile, allowing the planning of the restoration to be accomplished in a few minutes [9].

Among the methods of processing ceramic restorations without a doubt, milling using CAD/CAM technology seems to fit better when they propose to work with more practicality in a shorter clinical time.The production of the prosthetic parts begins through pre-fabricated blocks industrially milled in a milling unit. The LD glass ceramic (IPS e.max CAD, IvoclarVivadent, Barueri, $\mathrm{SP}$, Brazil) was designed for the CAD/CAM processing technology [10]. 
The concept of minimally invasive dentistry with preparation preferably at the enamel level, increase efficiency in adhesive cementation.The minimally invasive preparations should remain in enamel and consist of adjusting the tooth to receive the ceramic restoration preserving to the maximum the healthy dental structure, necessitating a simpler, more intuitive preparation [11].

The central incisors are the most dominant teeth in the smile, therefore, that the relation width $\mathrm{x}$ height is essential in the reproduction of the new architecture of the smile.The maintenance of the aesthetic predictability achieved in the diagnostic mock-up when applying the aesthetics concepts, following the checklist, tested and approved by the patient, is extremely important in the stage of processing of scanning of the restorations [12].

Therefore, the CAD / CAM technology provides the technical possibility of ceramic laminates, where one of the methods of making is the correlation technique, where the functional model is initially scanned to obtain the digital information of the preparation of mockup in the mouth. Through the correlation of the models the software has the final shape of the ceramic restorations quickly. The digital restorations are adjusted by contouring and then milled using the ceramic system selected with block in color and transparency previously determined by the integrated planning [13].

Digital technology is rapidly emerging and has introduced many new possibilities in dental practice because of the different advantages that the system offers. So, the aim of this case report is to present the esthetic results of multiple LD ceramic veneers manufactured with CAD/CAM using the correlation mode associated with the biogeneric mode.

\section{Case Report}

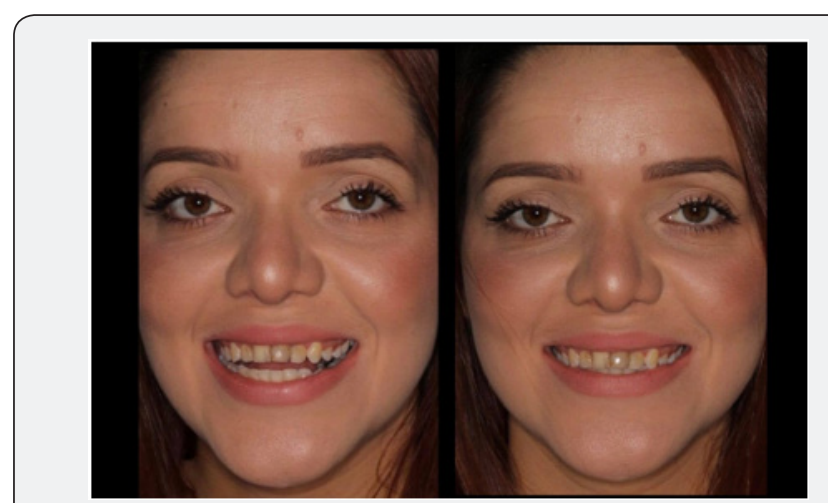

Figure 1: Initial clinical situation.

Patient, female, 25 years old, sought the ABO Taguatinga-DF, Brazil's clinic, the patient was not satisfied with the color and shape of her teeth, the smile aspect and lack of harmony between the lips and the teeth (Figure 1). She complaint that she was embarrassed to smile at work and at socially, demonstrating a desire for teeth that look younger and white in color. On examination of the smile the exposure of the gingival margin was observed in some regions and disharmonic restorations (Figure 2).After clinical and radiographic examinations and obtaining initial impressions, it was determined that the patient had a low caries risk and did not have active caries lesions or signs of periodontal disease. The esthetic treatment plan alternatives were discussed with the patient, including dental bleaching and gingivectomy, followed by ceramic laminates.

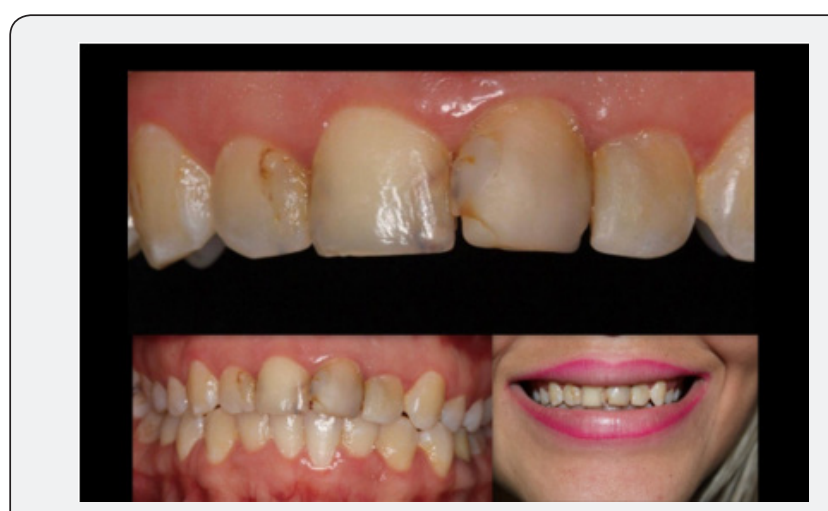

Figure 2: Close up of disharmonic restauration.

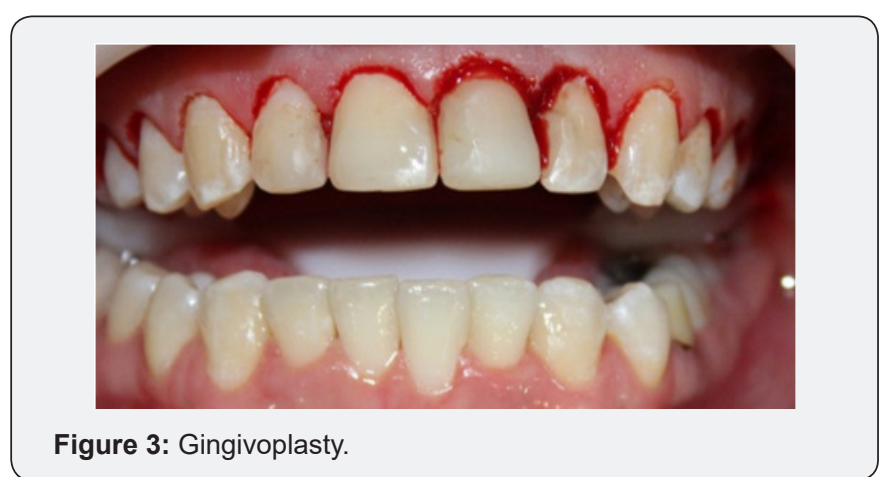

Figure 3: Gingivoplasty.

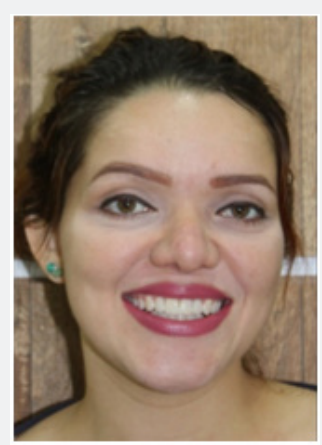

Figure 4: Frontal view of mock-up provisionalization.

Clinical procedures began with gingivoplastywith Thera Lase Surgery, upper and down arches (Figure 3). Then, a mock-up was carried out in composite resin to evaluation of size and shape and demonstration to patient (Figure 4). The mock-up is useful tool that allows showing the rehabilitation planning for patients with high aesthetical expectations before proceeding with the preparation step. The preliminary mock-up was performed using bisacrylic resin (Protemp 4, 3M ESPE) to simulate the result, to evaluate the size and shape and to support the patient and the professional to determine the ideal wid. These provisional veneers were tried 


\section{Advances in Dentistry \& Oral Health}

in for marginal integrity, functionality, occlusion, esthetics, and patient satisfaction. Then, the dental preparation was performed according to the technique (Figure 5).

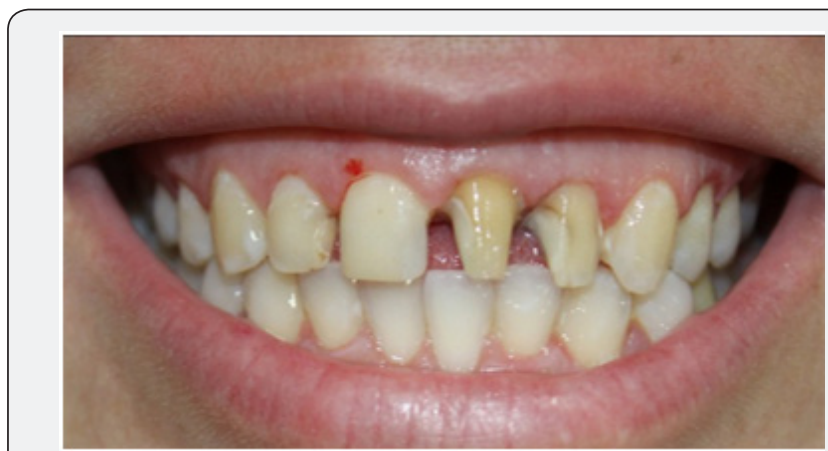

Figure 5: Finished tooth preparations.

Static and dynamic dentofacial aspects were evaluated, considering lip line and maxillary teeth exposure following the preparations a small diameter retraction cord was placed in the bottom of the sulcus to obtain an adequate gingival displacement (number 000 Ultrapak, Ultradent Inc.). The facial aspects of the patient are considered and viewed when the virtual teeth are designed. This procedure can produce a design that closely represents the definitive smile for patient evaluation. Subsequently, the intraoral scanner is also used to make the digital impression of the prepared teeth, to process the restorations' in a computer-aided design and computer-aided manufacturing (CADCAM) system.

An optical impression was made with the digital camera of the CEREC acquisition unit (Omnicam CEREC Sirona Bensheim, Germany) which has an accuracy of $19 \mu \mathrm{m}$, comparable to extra high-precision oral scanners[9]. Optical images of the antagonist teeth were also taken, and the bite registration was recorded with buccal scanning technique. In this technique, optical bite registration images were taken from buccal direction with the teeth occluded in maximum intercuspal position. The buccal bite registration image was dragged with the mouse approximately to the corresponding parts of the preparation and antagonist models. The software then recognized similar surfaces and automatically articulated the models in maximum intercuspal position. Then the preparation of 10 laminates color BL4 block HT in porcelain EMAX CAD by the system CEREC 4.4 (Figure 6 \&7).

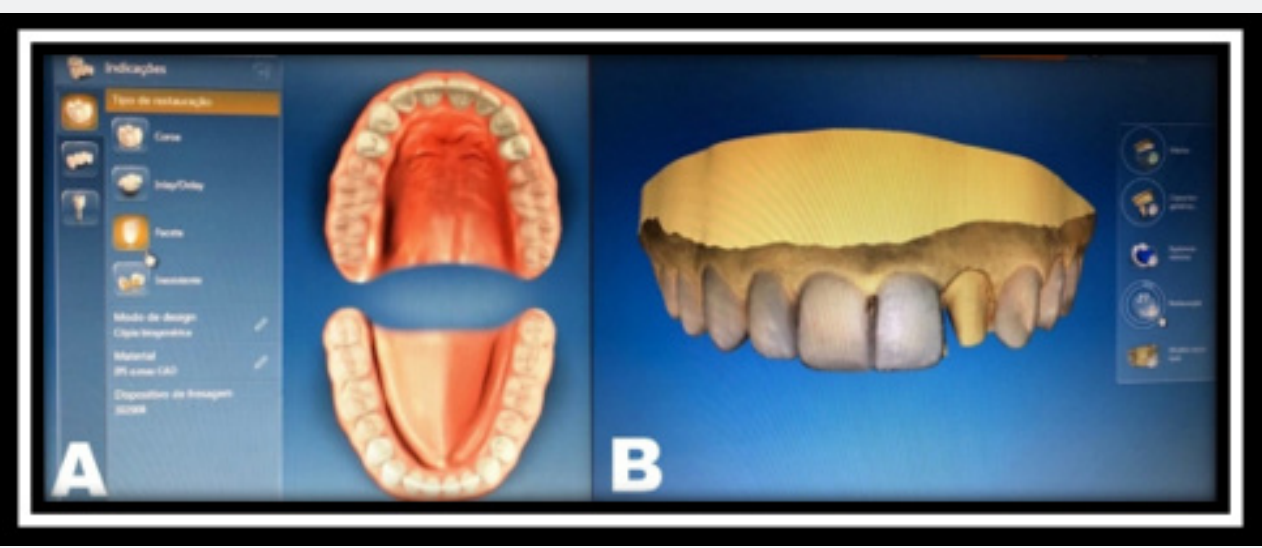

Figure 6

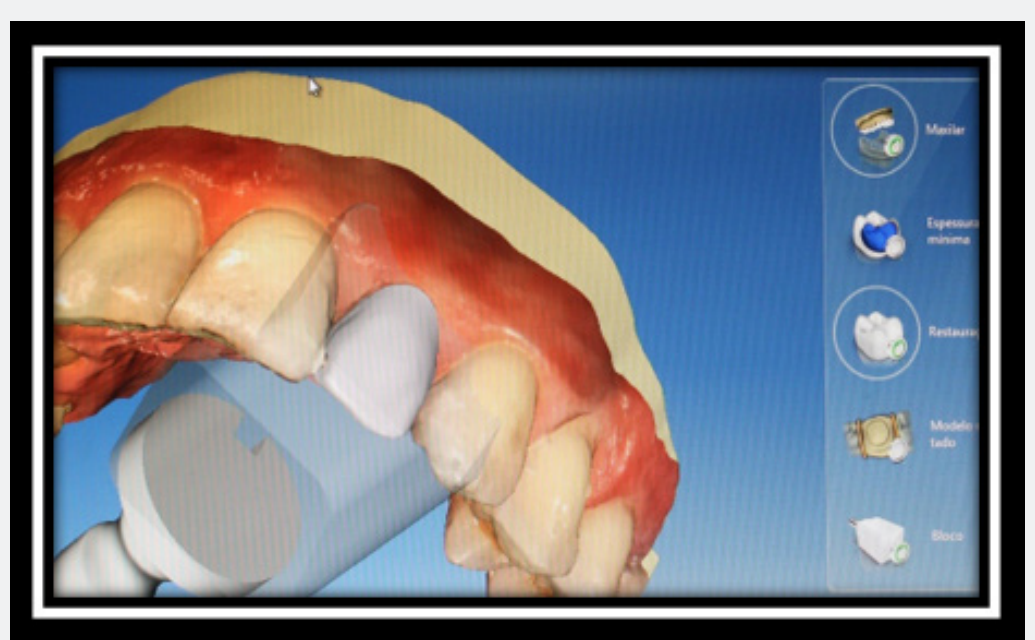

Figure 7: Supplement. 
Prior to cementation, the marginal adaptation, interproximal contacts, and occlusion of the laminates ceramics were checked. The color evaluation was tested with low-, medium-, and highvalue try-in paste of the Variolink Veneer cement (IvoclarVivadent).

Once the patient approved the restorations, the laminate veneers were prepared for bonding according to manufacturer's instructions. The internal areas of the laminate veneers were treated with $5 \%$ hydrofluoric acid etching gel conditioned (Power C Etching 5\%, BM4, Brasil Materials e Instrumentals LTDA, Palhoca, Santa Catarina, Brazil) for 60 s and cleaned using a water spray. After the restorations were dried for 20s, a silane coupling agent (Monobond S, IvoclarVivadent, Liechtenstein) was applied to the internal surfaces of all veneers and airdried for $5 \mathrm{~s}$. Then using a coat of adhesive Single Bond (3M) by scrubbing for 20 seconds and allowing the primer to react on the surface for 40 seconds, according to the respective manufacturers' instructions and left uncured (Figure 8).

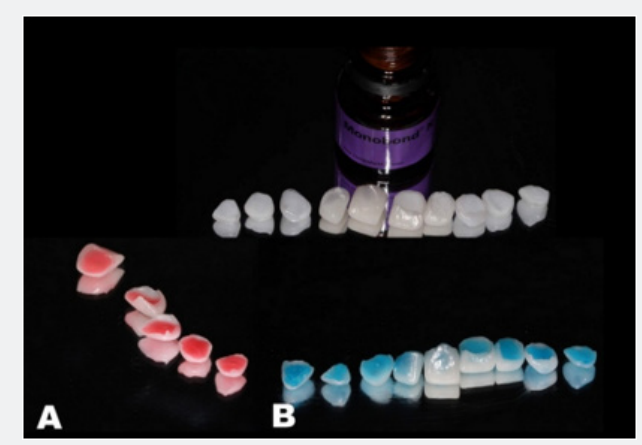

Figure 8: Surface treatment with $5 \%$ hydrofluoric acid for 20 seconds and $37 \%$ phosphoric acid. Silane application.

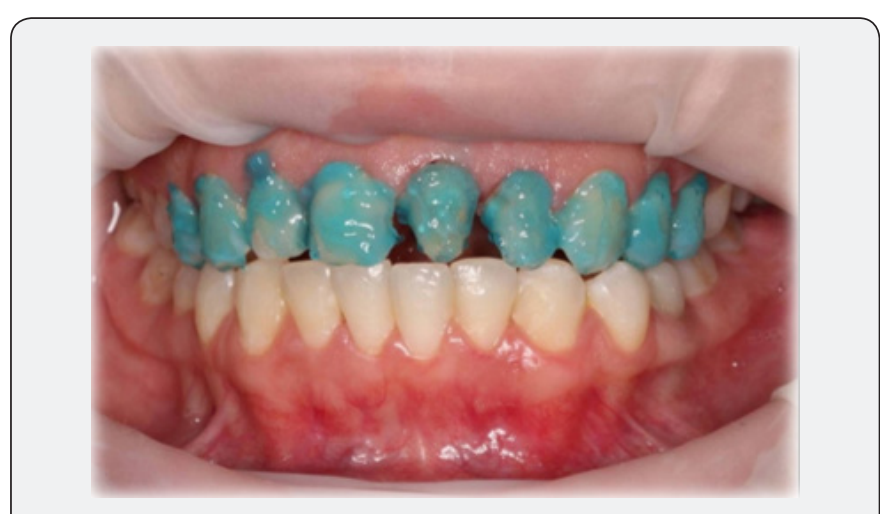

Figure 9: Enamel etching with $37 \%$ phosphoric acid application.

The prepared tooth surfaces were etched with 37\% Total Etch phosphoric acid (IvoclarVivadent) and rinsed after 15 seconds with an air water spray (Figure 9). Excess water was removed by gently blowing air.Gently blown air was used for solvent evaporation. The adhesive was not light cured. Then, the light-cured Variolink Veneer resin cement (Ivoclar-Vivadent) was placed on the intaglio surface of the veneer and seated into position. The excess resin cement was removed, and the veneers were light cured for 40 seconds from each of the dental surfaces. Then, LED light curing was performed on the facial, incisal, and palatal sides on each side (Valo, Ultradent Products Inc. South Jordan, Utah, USA) at $1,000 \mathrm{~mW} / \mathrm{cm} 2$. The occlusion was checked and adjusted, and the case was finalized (Figure10).

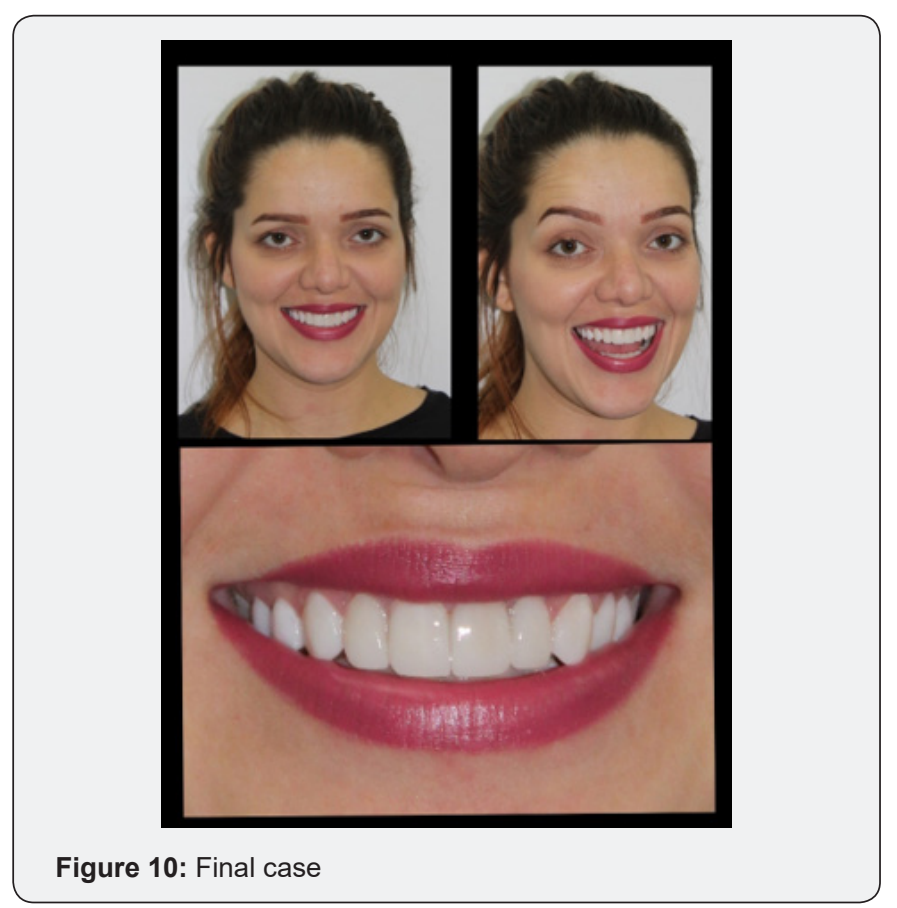

\section{Discussion}

The expectation of an esthetically harmonious smile increases the level of difficulty when treating patients. Laminate veneers stand out as a treatment option for cosmetic rehabilitation in clinical practice, as they are a more conservative procedure and mimic dental structures [5].

The classical molding techniques with subsequent obtaining of models of work in plaster still represent the gold standard in the process of manufacturing fixed restorations on teeth. However, there are some concessions inherent to conventional techniques that spend a lot of time, with an extensive need for highly specialized labor, logistics, time and equipment [1].

Recently, manufacturing materials, techniques, and office time have been simplified by digital dentistry and the introduction of stronger glass infiltrated ceramics. With the introduction of these ceramics, especially pressed lithium disilicate (LD), laminate veneers can be made using either the conventional lost-wax technique or by designing a prosthetic structure on a computer, followed by its manufacture in a milling machine [10].

The computer-aided design/computer-aided manufacturing (CAD/CAM) system optimizes the fabrication of prosthetic structures, reducing chairside time and promoting good esthetic results[14]. The current Cerec technology enables a very friendly user interface, because thanks to the evolution of the equipment and mainly to the data manipulation program, with a little training, the professional is able to produce a high quality Cerec restoration 
as demonstrated in one study with dentistry students with little prosthesis experience who attended a two-day course on Cerec.In this study the students fabricated inlays restorations that behaved clinically in a very satisfactory manner over a 3-year observation period [14].

However, a relevant aspect is the need for a tooth preparation smooth and defined, with visual access for image capture. Subgingival margin can't be copied by the capture system and the gingival tissue has to be retracted for proper capture [7].

Other aspect that should be observed is that, the restorations of ceramic with CAD-CAM system can be finished with polish or make-up and glaze, without prejudice to their superficial and intrinsic physical characteristics [15].

It should be noted that the progress in adhesive technologies has made possible to perform more conservative restoration techniques. However, beyond the evolution of resin cements in bonding, it's important to know that the different shades of resin cements may affect the final color of the ceramic veneers. Therefore, it is very important to select the matching color of tryin paste before the cementation [9].

The use of CAD/CAM to design a dental restoration is efficient and highly precise.In this case report laminate were fabricated using an all-digital workflow. In this way the case describes how a digital plan contributed to the achievement of a satisfactory and predictable esthetic outcome. A precise diagnosis and a comprehensive plan that considers facial and dentogingival individual aspects should be mandatory to predict the result of an oral treatment, especially when esthetics are involved [14].

CADCAM technology, coupled with a highly performance work protocol, can generate predictable results, optimizing clinical and laboratory logistics. This technology makes possible the execution of rehabilitation treatments in an optimized way without, however losing the quality while maintaining the initial aesthetic predictability. Furthermore, the use of these systems to manufacture porcelain veneers allows the dentist to control the time as well as color, contour, and form.

\section{References}

1. Miyazaki T, Hotta Y (2011) CAD/CAM systems available for the fabrication of crown and bridge restorations. Aust Dent J 56(s1): $97-$ 106.
2. Magne P, Hanna J, Magne M (2013) The case for moderate "guided prep" indirect porcelain veneers in the anterior dentition. The pendulum of porcelain veneer preparations: from almost no-prep to over-prep to no-prep. Eur J Esthet Dent 8(3): 376-388.

3. Akan E, Bagis B (2016) Midline diastema closure with partial laminate veneers: A case report. Balk J Dent Med 20(1): 59-62.

4. de Siqueira F, Cardenas A, Gruber YL, Kose C, Pupo YM, et al. (2017) Using CAD/CAM-Modified Correlation Mode to Produce Laminate Veneers: A Six-Month Case Report. Oper Dent 42(5): 139-147.

5. Passos L, Soares FP, Gallo M (2016) Esthetic Rehabilitation through Crown Lengthening Surgery and Conservative CAD/CAM Veneers: A Multidisciplinary Case Report. Case reports in dentistry p.07.

6. Almasri R, Drago CJ, Siegel SC, Hardigan PC (2011) Volumetric misfit in CAD/CAM and cast implant frameworks: a university laboratory study. Journal of Prosthodontics 20(4): 267-274.

7. Abduo J, Bennamoun M, Tennant M, McGeachie J (2016) Impact of digital prosthodontic planning on dental esthetics: Biometric analysis of esthetic parameters. The Journal of prosthetic dentistry 115(1): 5764.

8. Frankenberger R, Krämer N, Appelt A, Lohbauer U, Naumann M, et al. (2011) Chairside vs. labside ceramic inlays: effect of temporary restoration and adhesive luting on enamel cracks and marginal integrity. Dental materials 27(9): 892-898.

9. Coachman C, Gurel G, Calamita M, Morimoto S, Paolucci B, et al. (2014) The influence of tooth color on preparation design for laminate veneers from a minimally invasive perspective: case report. Int J Periodontics Restorative Dent 34(4): 453-459.

10. Çavuşoğlu Y, Sahin E, Gürbüz R, Akça K (2011) Fatigue resistance of 2 different CAD/CAM glass-ceramic materials used for single-tooth implant crowns. Implant Dent 20(5): 374-378.

11. Pereira LM, Resende CCD, Tavares LN, Maruki AY, Prudente MS (2017) Esthetic Rehabilitation of Smile with Minimally Invasive Preparations and Thin Laminate Ceramic Veneers Using Chairside CAD/CAM System. J Dent Health Oral Disord Ther 8(1): 00267.

12. Re D, Augusti G, Amato M, Riva G, Augusti D (2014) Esthetic rehabilitation of anterior teeth with laminates composite veneers. Case reports in dentistry p. 9.

13. Martins AV, Albuquerque RC, Santos TR, Silveira LM, Silveira RR, et al. (2017) Esthetic planning with a digital tool: A clinical report. J Prosthet Dent 118(6): 698-702.

14. Lin WS, Zandinejad A, Metz MJ, Harris BT, Morton D (2015) Predictable Restorative Work Flow for Computer-Aided Design/Computer-Aided Manufacture-Fabricated Ceramic Veneers Utilizing a Virtual Smile Design Principle. Oper Dent 40(4): 357-363.

15. Fasbinder DJ, Neiva GF (2016) Surface evaluation of polishing techniques for new resilient CAD/CAM restorative materials. J Esthet Restor Dent 28(1): 56-66. 
This work is licensed under Creative Commons Attribution 4.0 License DOI: 10.19080/ADOH.2018.09.555769
Your next submission with Juniper Publishers will reach you the below assets

- Quality Editorial service

- Swift Peer Review

- Reprints availability

- E-prints Service

- Manuscript Podcast for convenient understanding

- Global attainment for your research

- Manuscript accessibility in different formats ( Pdf, E-pub, Full Text, Audio)

- Unceasing customer service

Track the below URL for one-step submission https://juniperpublishers.com/online-submission.php 